Article

\title{
Viscoelastic Rheological Behaviors of Polypropylene and LMPP Blends
}

\author{
Feichao Zhu ${ }^{1,2,+}$, Sohail Yasin ${ }^{3, *(D)}$ and Munir Hussain ${ }^{3,+} \mathbb{D}_{\mathbb{D}}$ \\ 1 Department of Textile Engineering, College of Textile Science and Engineering, Zhejiang Sci-Tech University, \\ Hangzhou 310027, China; zhufeichao@zstu.edu.cn \\ 2 Zhejiang Provincial Key Laboratory of Industrial Textile Materials and Manufacturing Technology, \\ Zhejiang Sci-Tech University, Hangzhou 310027, China \\ 3 MOE Key Laboratory of Macromolecular Synthesis and Functionalization, Department of Polymer Science \\ and Engineering, Zhejiang University, Hangzhou 310027, China; munir88@zju.edu.cn \\ * Correspondence: soh.yasin@gmail.com \\ + These authors contributed equally.
}

check for updates

Citation: Zhu, F.; Yasin, S.; Hussain, M. Viscoelastic Rheological Behaviors of Polypropylene and LMPP Blends. Polymers 2021, 13, 3485. https:// doi.org/10.3390/polym13203485

Academic Editors: Eduardo Guzmán and Alexander Malkin

Received: 17 August 2021

Accepted: 1 October 2021

Published: 11 October 2021

Publisher's Note: MDPI stays neutral with regard to jurisdictional claims in published maps and institutional affiliations.

Copyright: (c) 2021 by the authors. Licensee MDPI, Basel, Switzerland. This article is an open access article distributed under the terms and conditions of the Creative Commons Attribution (CC BY) license (https:// creativecommons.org/licenses/by/ $4.0 /)$.
Abstract: Dynamic oscillatory shear testing is used to investigate polymeric viscoelastic behaviors. Small and large amplitude oscillatory shear tests are the canonical method for characterizing the linear and nonlinear viscoelastic behaviors of any polymeric material. With prominent and abundant work on linear viscoelastic studies, the nonlinear behavior is evasive in terms of generating infinite higher harmonics in the nonlinear regime. For this reason, intrinsic nonlinearities from large amplitude oscillatory shear (LAOS) studies have recently been used for insights on microstructural behaviors. This study is carried out for linear and nonlinear viscoelastic behavior with a main focus on LAOS of isostatic polypropylene (iPP) and relatively new low molecular weight and low modulus polypropylene-based polyolefin (LMPP) blends. The morphological results showed reduced spherulitic crystal nucleus size and increased distribution in blends with increasing LMPP. The blends showed subtle linear viscoelastic responses with strong nonlinear mechanical responses to variant strain and stress compared to pure iPP. The intracycle strain thickening and intracycle strain stiffening of high-content LMPP blends were comparatively dominant at medium strain amplitudes.

Keywords: LMPP; polypropylene; LAOS; rheology; Lissajous

\section{Introduction}

Isostatic polypropylene (iPP), classified as an important polymer, is often restricted due to relatively low toughness and high thermal expansion coefficients [1]. Blending and polymeric modifications are required to improve the properties. Blending of iPP with a low molecular weight and low modulus (LMPP) in small amounts [2-4], has been reported to produce finer and softer fiber products, such as nonwovens. LMPP is a relatively new polypropylene-based polyolefin developed by Idemitsu Petrochemical, Ltd., posing variant properties associated with conventional polypropylene. High crystallinity and melting point, which is attained using metallocene catalyst, reinforces the stereoregularity of the polymer [5]. Polyolefin elastomers provide elasticity, cohesive strength and adequate melt flow rate, yet show immiscibility, even when having similar monomer chemistry [6]. However, the properties only develop slowly after the application, as the high crystallinity is critical to bond stability over time [7]. Moreover, iPP and elastomer blends are heterogeneous at low temperatures, thus the properties are dependent on shape and size distribution of polyolefin spherulites of the blends [8]. In terms of improved iPP products, morphology and viscoelastic properties of the blends are essentially important when blended with newer polymers. Moreover, determination of the rheological properties is considered crucial for the fundamental understanding of blends processability $[9,10]$.

iPP, being thermoplastic polymer, requires high processing temperatures to reach appropriate melt viscosity. To analyze viscosity, a capillary rheometer is considered suitable 
for measuring rheological properties of iPP blends at high temperatures and with wide shear rate ranges. It provides easy to handle high viscous melts at high temperatures and is industrially applicable by being similar to actual processing extrusion or injection molding with the same shear rates and flow geometry [11,12]. Immiscibility due to low entropy of mixing, phase separation and low interaction with each other are known hurdles while blending. Indeed, the properties of the blends are dependent on physical properties, including the morphology and the chemistry of the polymers, showing their interactions. Degree of polymeric dispersion/distribution and tendency of phase separation can be distinguished rheologically. This work provides effects of LMPP in iPP blends morphology accompanied by viscoelastic characterization. Viscoelastic characterization with dynamic rheological analysis of polymeric materials is important [13-15] for prior final designing and processing of any product $[16,17]$. In viscoelastic characterization, application of a large amplitude oscillatory strain (LAOS) marks non-sinusoidal stress responses, acknowledged as nonlinear response. Later, stress decompositions by Fourier transform [18] on time domain stress response, resulting power spectrum, are frequently applied for the interpretation of LAOS data [19-21]. LAOS measurements have shown higher sensitivity to instantaneous structural changes in polymeric solutions and melts [1,14]. Qualitative data from LAOS is graphically presented with Lissajous plots, where stress and strain are shown as a function of elastic and viscous responses. Next, the intercycle and intracycle responses focusing on elastic and viscous nonlinearities are investigated at different strain amplitudes.

\section{Materials and Methods}

The isotactic polypropylene (iPP), s2040 (Ziegler-Natta) with a molecular weight (MW) $2.0 \times 10^{5} \mathrm{~g} / \mathrm{mol}$ was provided by SECCO Petrochemical Ltd., Shanghai, China. The LMPP (s901) was provided by Idemitsu Kosan Co, Ltd. Tokyo, Japan, with MW $1.3 \times 10^{5} \mathrm{~g} / \mathrm{mol}$. The characterization data for the material used are presented in Table 1 . The polymeric materials used in this work were of laboratory grade samples. Melt flow rate index (MFR) was $50 \mathrm{~g} / 10 \mathrm{~min}$ for LMPP. The mixing ratios of iPP/LMPP by (wt/wt): $100 / 0,95 / 5,90 / 10,85 / 15,80 / 20,75 / 25$, were prepared and defined as $5 \%, 10 \%, 15 \%, 20 \%$ and $25 \%$ LMPP blends. The morphological effects of iPP/LMPP blends were investigated by comparing $100 \%$ iPP and 100\% LMPP.

Table 1. Ingredients used for the blends.

\begin{tabular}{cccccccc}
\hline Materials & Type & Catalyst & ${ }^{\mathbf{1}} \mathbf{M W ~ ( g / m o l )}$ & ${ }^{2}$ MW/MN & Density $\left(\mathbf{k g} / \mathbf{m}^{\mathbf{3}}\right)$ & MFR $(\mathrm{g} / \mathbf{1 0} \mathbf{~ m i n})$ & ${ }^{3}$ TM $\left({ }^{\circ} \mathbf{C}\right)$ \\
\hline iPP & Isotactic & Ziegler Natta & 200,000 & 3.5 & 900 & 36 & 165.5 \\
LMPP & Atactic & Metallocene & 130,000 & 2 & 870 & 50 & 79.1 \\
\hline
\end{tabular}

${ }^{1} \mathrm{MW}$ : molecular weight; ${ }^{2} \mathrm{MW} / \mathrm{MN}$ : molecular weight/molecular number; ${ }^{3} \mathrm{TM}$ : melting temperature.

Mechanical blends of iPP/LMPP at the compositions of interest were prepared with the help of a mechanical blender by Giant Co., Ltd. Nanjing, China and mixed for $2 \mathrm{~min}$ at room temperature. Melt-blended samples of the homopolymers with various compositions were prepared by a twin-screw extruder (TSE-30A Ruiya extrusion system Co., Ltd., Nanjing, China) with an $\mathrm{L} / \mathrm{D}=40$, and the processing speed of extruder was $60 \mathrm{rpm}$ at $210^{\circ} \mathrm{C}$.

Polarized optical and light microscope (POM/PLM) was used to characterize the growth of iPP/LMPP spherulites and crystals morphology. For that, a small piece of sample was cut and compressed manually in a compression machine at $\sim 30$ bars. The samples were placed on a hot stage and preheated at $25^{\circ} \mathrm{C}$ to $200^{\circ} \mathrm{C}$ at a rate of $50{ }^{\circ} \mathrm{C} / \mathrm{min}$ for $2 \mathrm{~min}$ until the samples were melted. Reaching $200^{\circ} \mathrm{C}$, the samples were cooled at $120^{\circ} \mathrm{C}$ at a rate of $50{ }^{\circ} \mathrm{C} / \mathrm{min}$ for $15 \mathrm{~min}$ isothermal temperature. At a temperature of $120^{\circ} \mathrm{C}$, POM images were captured. A Leica DM2700P transmission/reflection polarizing microscope (Beijing, China) was used. Hot-pressed blends into tablets $(\sim 0.2 \mathrm{~mm})$ were observed at room temperature after cooling and crystallization.

The capillary rheology of the blends was carried out at three varying temperatures: 190,200 and $210^{\circ} \mathrm{C}$. The blends were added to the barrel after two preloads ( $0.3 \mathrm{MPa}$ ) and 
two heat-rheological tests (total $5 \mathrm{~min}$ ) later. The shear rate range was $100-6000 \mathrm{~s}^{-1}$, and capillary diameter $1 \mathrm{~mm}$ with length to diameter ratio $\mathrm{L} / \mathrm{D}=16$. A die of zero shear stress was used. Dynamic linear rheological responses of the blends at $150^{\circ} \mathrm{C}$ were measured on a strain-controlled rheometer (ARES-G2, TA Instruments, New Castle, DE, USA) with a plate geometry of $25 \mathrm{~mm}$ in diameter. Angular frequency $(\omega)$ sweep tests, from $\omega=100 \mathrm{rad} / \mathrm{s}$ to $0.01 \mathrm{rad} / \mathrm{s}$ at $\gamma=0.5 \%$, were conducted. Similarly for LAOS, oscillatory data were obtained in parallel plate geometry of $25 \mathrm{~mm}$ in diameter at $150{ }^{\circ} \mathrm{C}$ at frequency $(\omega)$ $1.0 \mathrm{rad} \mathrm{s}^{-1}$ from the same rheometer. Rheological response characterization of pure LMPP was restricted for its low melting temperature compared to iPP, thus it was not included in the analysis. The strain amplitudes $\left(\gamma_{0}\right)$ were set from $0.1 \%$ to $1000 \%$. In the linear regime, when a sinusoidal strain $\gamma(t)=\gamma_{0} \sin (\omega t)$ is applied at a given $\gamma_{0}$ to the blends, a sinusoidal stress $\sigma(t)=\sigma_{0} \sin (\omega t+\delta)$ with stress amplitude $\sigma_{0}$ and phase angle $\delta$ is generated [14,22-24]. Decomposition of $\sigma(t)$ into $\sigma(t)=\sigma^{\prime} \sin (\omega t)+i \sigma^{\prime \prime} \cos (\omega t)$ yields the first-order harmonics, i.e., elastic $\left(G^{\prime}=\sigma^{\prime} / \gamma_{0}\right)$ and viscous moduli $\left(G^{\prime \prime}=\sigma^{\prime \prime} / \gamma_{0}\right)$, which are used for linear viscoelasticity characterization. Here $\sigma^{\prime}$ and $\sigma^{\prime \prime}$ are in- and out-of-phase stress responses, respectively. Whereas, in nonlinear regimes, the higher order harmonics depending on $\gamma_{0}$ and $\omega$ [25] become more essential [26]. For decomposing stress into $\mathrm{n}^{\text {th }}$-order harmonics $\mathrm{G}_{n}^{\prime}\left(\gamma_{0}, \omega\right)$ and $\mathrm{G}^{\prime \prime}{ }_{\mathrm{n}}\left(\gamma_{0}, \omega\right)$, Fourier series [19], $\sigma\left(\mathrm{t} ; \omega, \gamma_{0}\right)=\gamma_{0} \Sigma_{\mathrm{n}, \mathrm{odd}}$ $\left[\mathrm{G}^{\prime}{ }_{n}\left(\omega, \gamma_{0}\right) \sin (\mathrm{n} \omega \mathrm{t})+\mathrm{i} \mathrm{G}^{\prime \prime}{ }_{n}\left(\omega, \gamma_{0}\right) \cos (\mathrm{n} \omega \mathrm{t})\right]$, is used. Lissajous plots for $\sigma^{\prime}$ against $\gamma$ and $\sigma^{\prime \prime}$ against $\dot{\gamma}$ and Fourier transform spectrum are evaluated to quantify the nonlinearity of given iPP/LMPP blends [27].

\section{Results}

POM was used to investigate the growth of the spherulite in iPP/LMPP blends. The spherulites of the blends were characterized at $120{ }^{\circ} \mathrm{C}$ for $15 \mathrm{~min}$ isothermally. From Figure 1, influence of LMPP on the size, structure and types of spherulite crystals is visible, while pure LMPP appeared with no spherulites, indicating the crystallinity being very low $[3,28]$. On the other hand, the pure iPP contains many spherulites that show higher crystallinity than LMPP. However, spherulites with increased LMPP content to iPP sharpen the pellets and round the shape with clear boundaries with bright light birefringence within the spherulites. The crystalline morphology of iPP/LMPP blends can be seen in the polarized light microscope image in Figure 2. It was found that the spherulites of pure iPP were obvious with large size $(\sim 50 \mu \mathrm{m})$ and high crystallinity, while being low for pure LMPP with no obvious spherulites. Addition of LMPP to iPP reduces the crystal nucleus size affecting iPP spherulites in high dispersion/distribution. The molecular chain in LMPP enters into the iPP macromolecular chain, which alters the iPP crystallization process [2-4]. During the crystallization of pure iPP, the spherulites grow and the compression inhibits the increase of the diameter. The addition of LMPP may have a dilution effect and may act as a plasticizer, increasing space between spherulites of the blends till the content increased to $15 \%$. Reaching the threshold, the size of spherulites appeared to be decreasing in 25\% LMPP blend (Figure 2g). Similarly, we found the degree of crystallinity of blends increases for $5-15 \%$ LMPP contents and then progressively decreases for $20-25 \%$ with possible diluent LMPP molecular suppression entanglement between the iPP molecules [3]. The mobility restriction of iPP molecules during the crystallization has also been reported for the decrease in crystallinity of iPP blends [3,29]. 

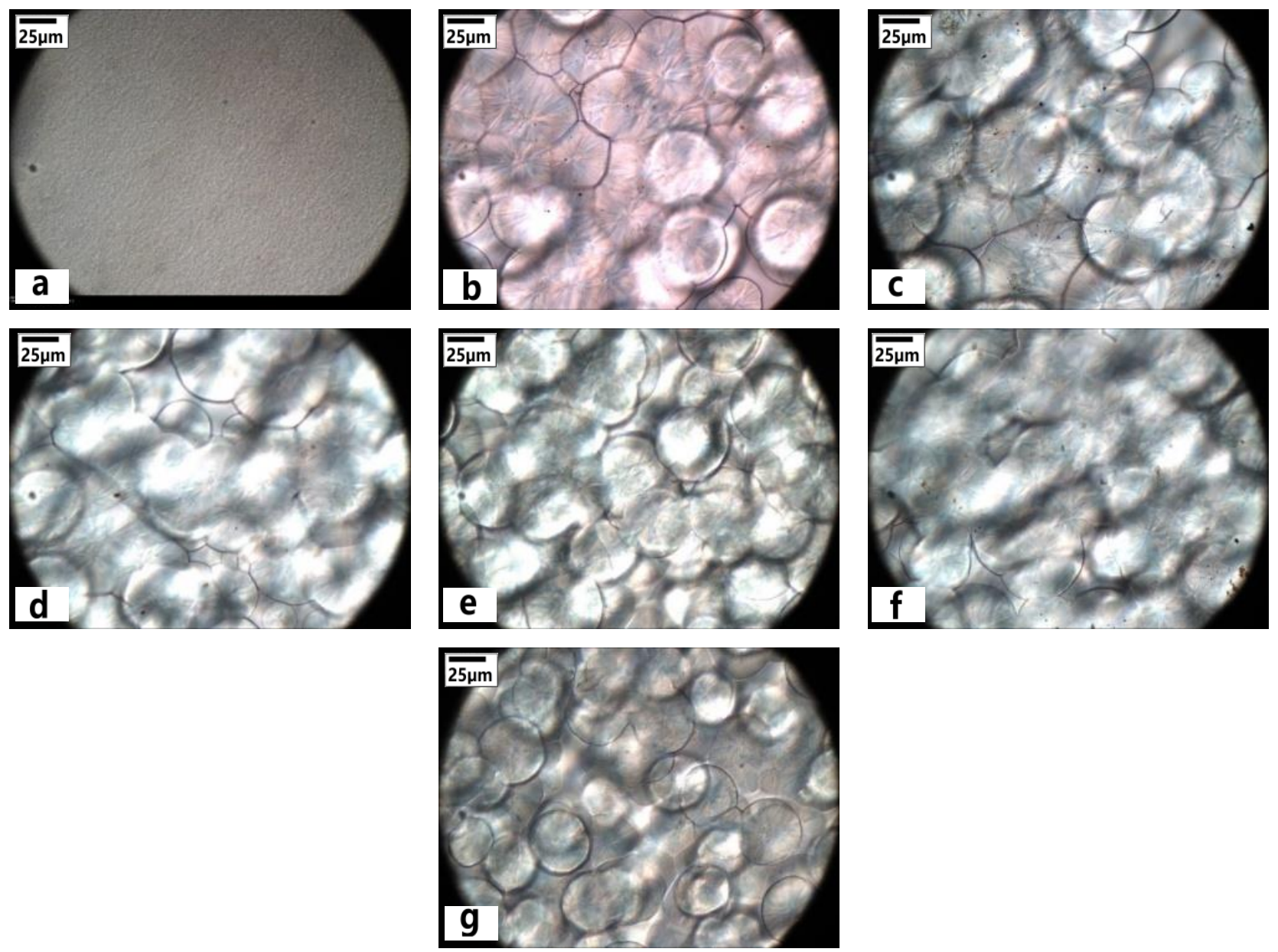

Figure 1. Morphology of spherulites for pure (a) LMPP, (b) iPP, (c) 5\%, (d) 10\%, (e) 15\%, (f) 20\% and (g) 25\% LMPP blends.
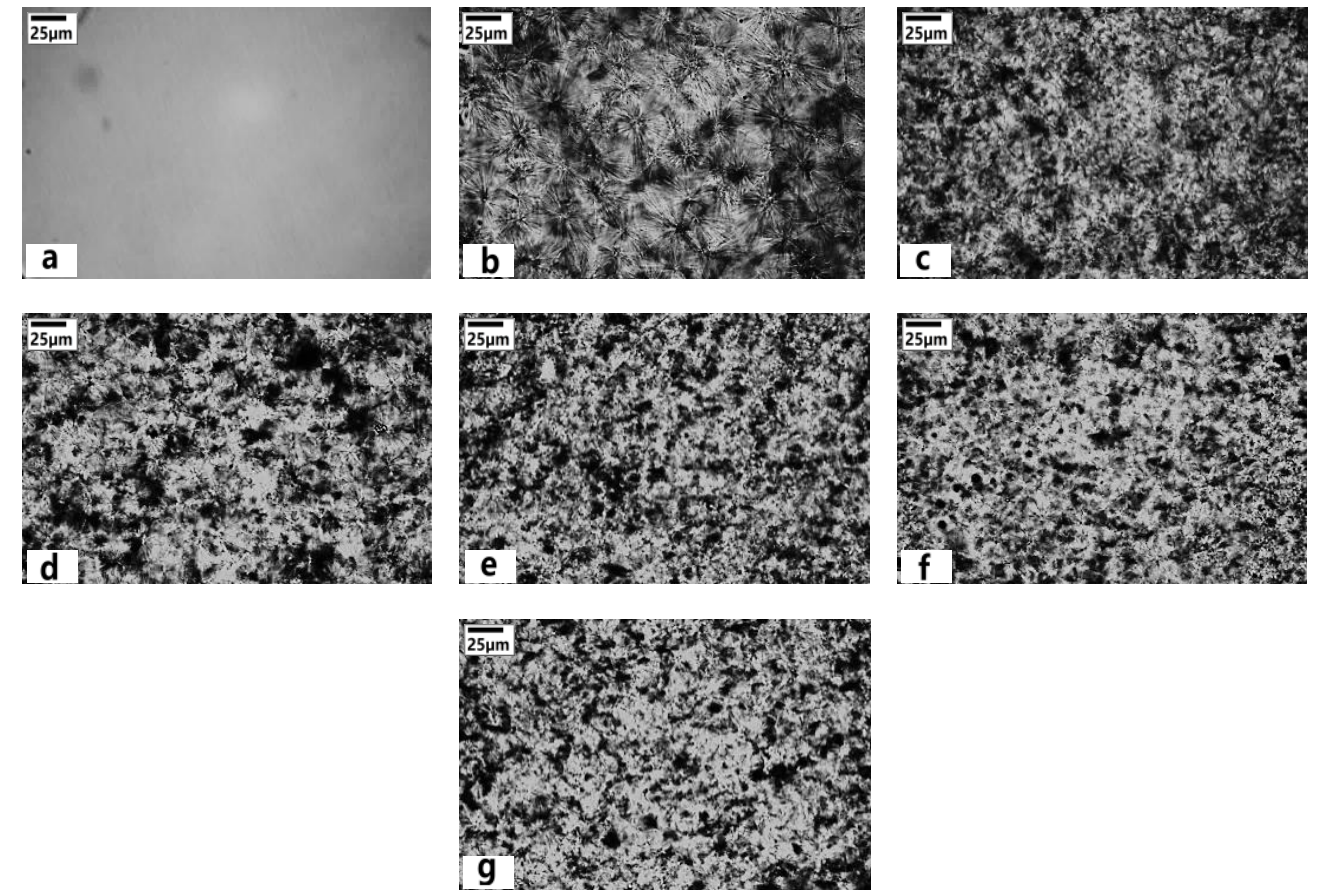

Figure 2. Morphology of pure (a) LMPP, (b) iPP, (c) 5\%, (d) 10\%, (e) 15\%, (f) 20\% and (g) 25\% LMPP blend crystals (adapted from [29]). 
Rheological evaluation differentiates interaction effects on polymer chains from phase separation, cross-linking network structure in the melt and incompatibility, providing information on the morphology evolution and reconfiguration of the network structure [30]. Capillary rheological behavior of the iPP and blends with different LMPP composition are shown in Figure 3. At shear rates near zero, the apparent viscosity of the melts slightly rose with increasing LMPP contents. At 5\% LMPP content, the viscosity is the lowest (Figure 3a); at 25\% the apparent viscosity is near to pure iPP 250 Pa.s. At lower shear rates, the viscosity of blends appears to be reducing down to 150 Pa.s.; this later improves slightly. Between 1000-3000 s ${ }^{-1}$, the decline is eased, reaching to 25 Pa.s. Similar behavior was seen in blends at higher temperatures, 200 and $210{ }^{\circ} \mathrm{C}$. The increasing temperatures and decreasing shear viscosity may be incurred because of enhanced macromolecule chain movement, also decreasing the apparent viscosity.
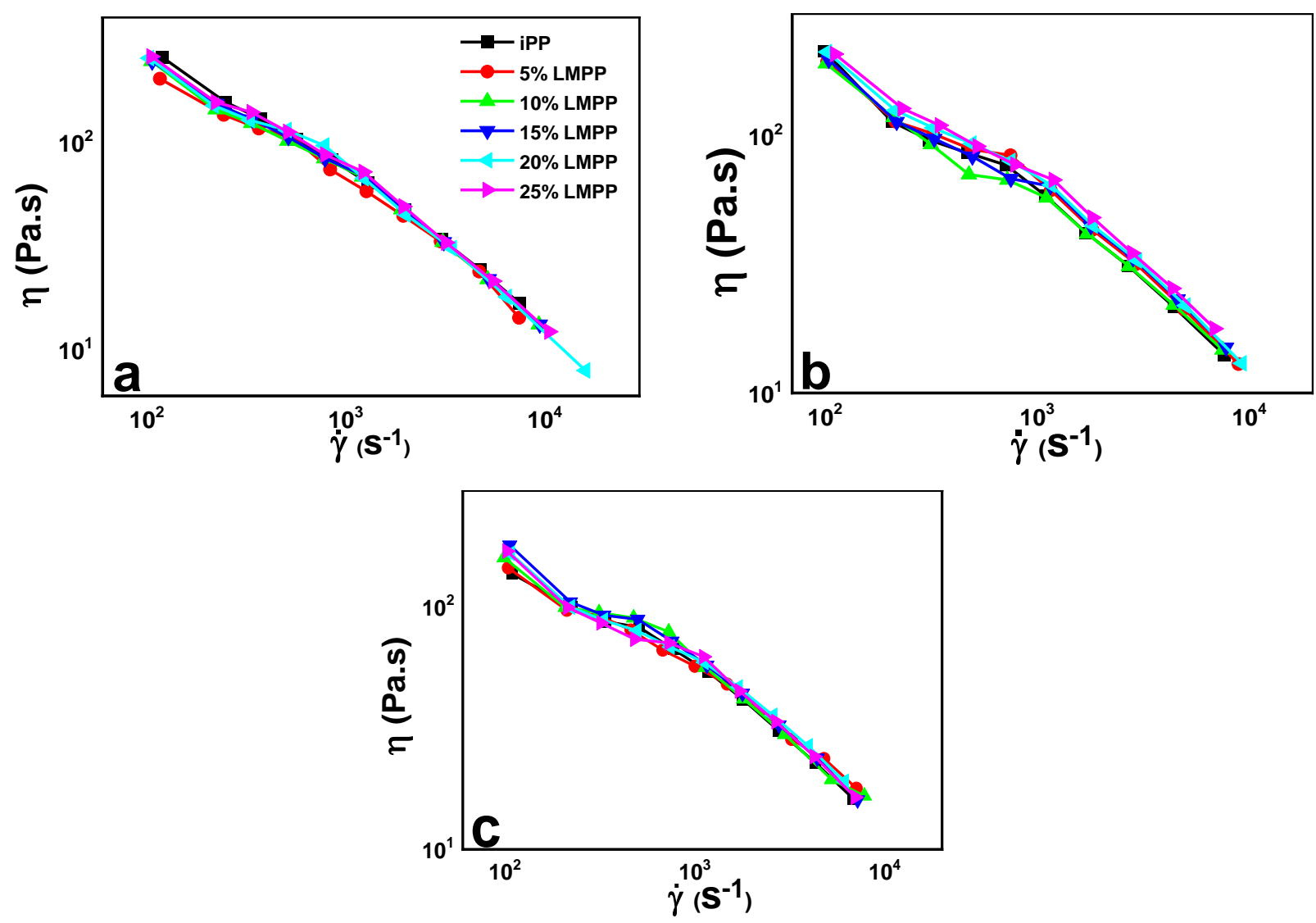

Figure 3. Capillary rheology plot of shear viscosity $(\eta)$ against shear rate $(\dot{\gamma})$ for pure iPP, LMPP and blends at (a) 190, (b) 200 and (c) $210^{\circ} \mathrm{C}$.

Similarly, in linear rheology of blends, storage modulus $\left(G^{\prime}\right)$ and loss modulus $\left(G^{\prime \prime}\right)$ as a function of frequency $(\omega)$ of iPP/LMPP blends showed slight changes compared to pure iPP; such behavior is known in cross-linked polymers [31,32]. The moduli $\mathrm{G}^{\prime \prime}>\mathrm{G}^{\prime}$ were dominant across the majority of $\omega$ range, which indicates the predominant viscous response of the blends and the pure iPP. Overall improved $\omega$ dependence of blends was seen more in $G^{\prime \prime}$ than $G^{\prime}$, while the loss tangent $(\tan \delta)$ showed arbitrary behavior at lower $\omega$ (Figure $4 \mathrm{c}$ ). The max. $\tan \delta$ was higher for pure iPP and the blends with $15 \%$ and $25 \%$ LMPP contents at lower $\omega$. 

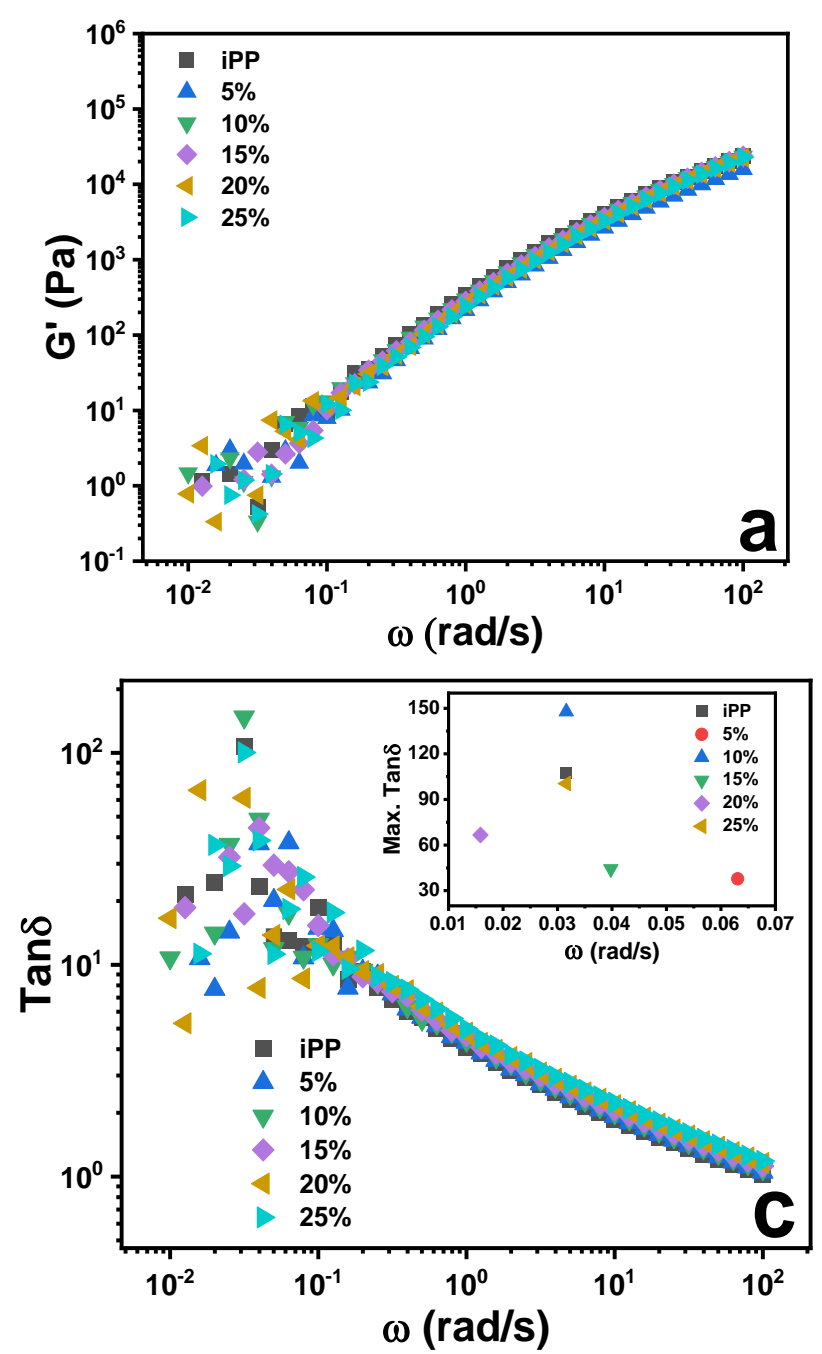
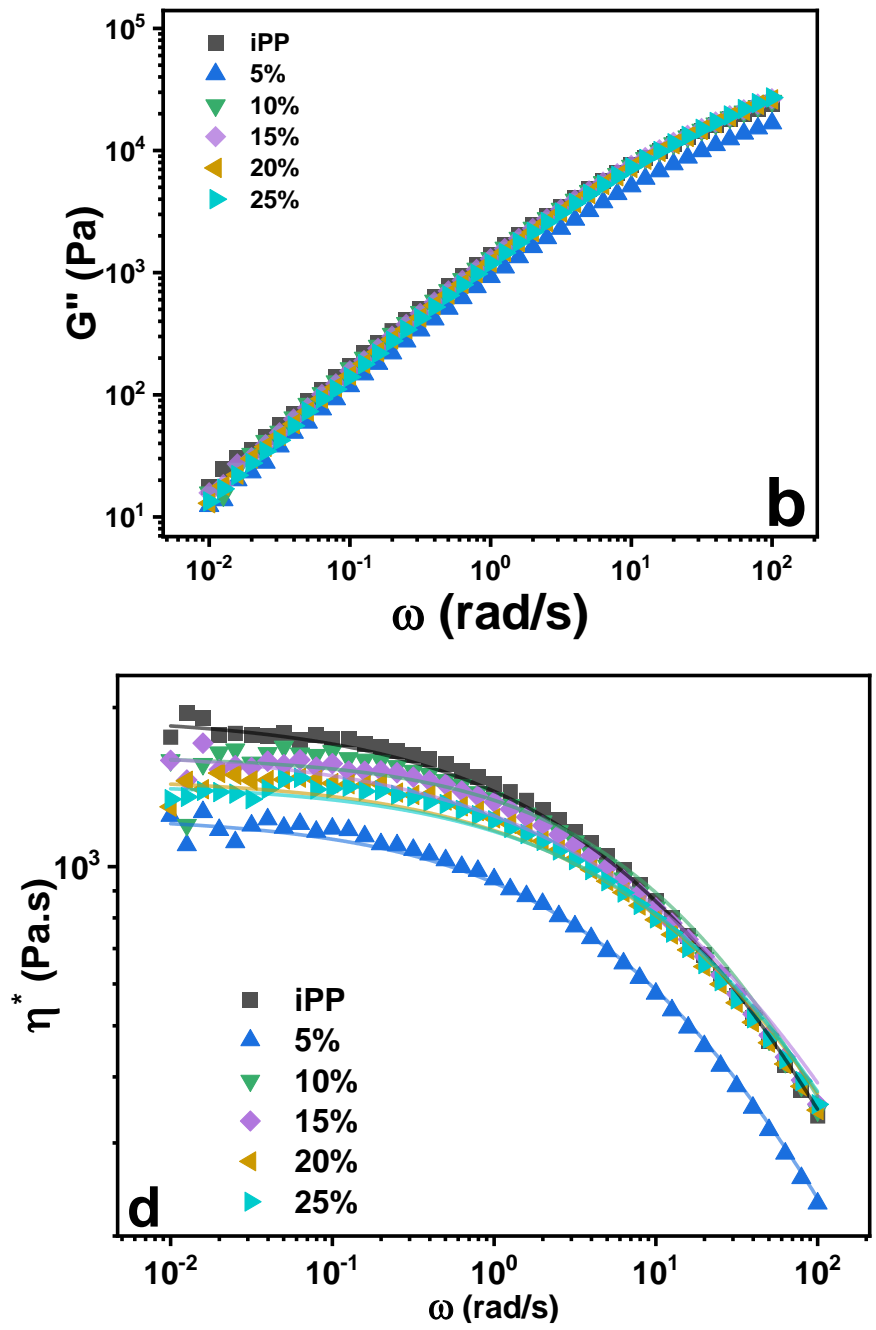

Figure 4. Dynamic rheological response for storage modulus $\left(G^{\prime}\right)(\mathbf{a})$, loss modulus $\left(G^{\prime \prime}\right)(\mathbf{b})$, loss tangent $(\tan \delta)$ with inset $(\max . \tan \delta)(\mathbf{c})$ and complex viscosity $\left({ }^{*} \eta\right)$, line 'fitted with Carreau-Yasuda model $(\mathbf{d})$ as a function of angular frequency $(\omega)$ at $\gamma=0.5 \%$ and $150{ }^{\circ} \mathrm{C}$ for pure iPP, LMPP and blends.

The complex viscosity $\left({ }^{*} \eta\right)$ of the pure iPP and LMPP blends is shown in Figure $4 \mathrm{~d}$; the data are fitted with the Carreau-Yasuda model [33], suggesting pure iPP, and all blends demonstrated shear-thinning behavior. Addition of lower $\mathrm{M}_{\mathrm{w}}$ polymer LMPP lowers the ${ }^{*} \eta$ of the blends compared to pure iPP, which may be ascribed to the better polymeric adhesion between them.

$G^{\prime}$ and $G^{\prime \prime}$ being linear viscoelastic moduli are not sufficiently enough to determine the material's responses, as the stress wave is no longer sinusoidal beyond the linear regime [34]. For that purpose, LAOS studies are carried out. Figure 5a shows the elastic Lissajous curves of the $\sigma$ against $\gamma_{0}$; at lower $\gamma_{0}$ the blends kept constant shape, with no response with the addition of LMPP. Whereas at higher $\gamma_{0}$ the blends kept the elliptical shape which widened reaching the rectangular shape, referring to the plastic characteristic of the materials $[14,22]$. Whereas from Figure $5 b$, at each chosen $\dot{\gamma}_{0}$ the shape of the Lissajous plot distorts as $\dot{\gamma}_{0}$ increases. It can be seen from Figure 5 that blends with $20 \%$ LMPP have the sharpest and clearest shape and boundaries at all $\gamma_{0}$ and $\dot{\gamma}_{0}$, ascribed to better physical polymeric interactions. 


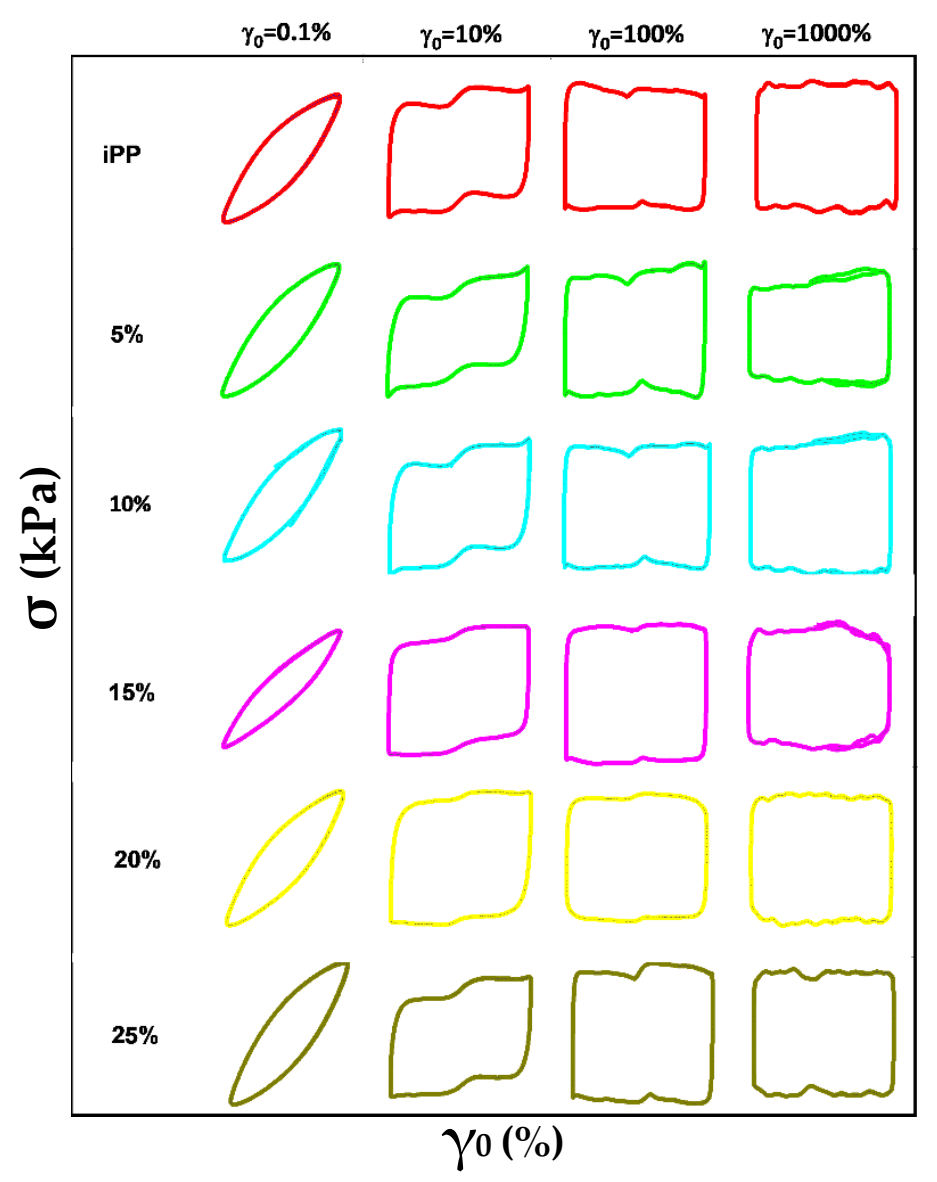

(a)

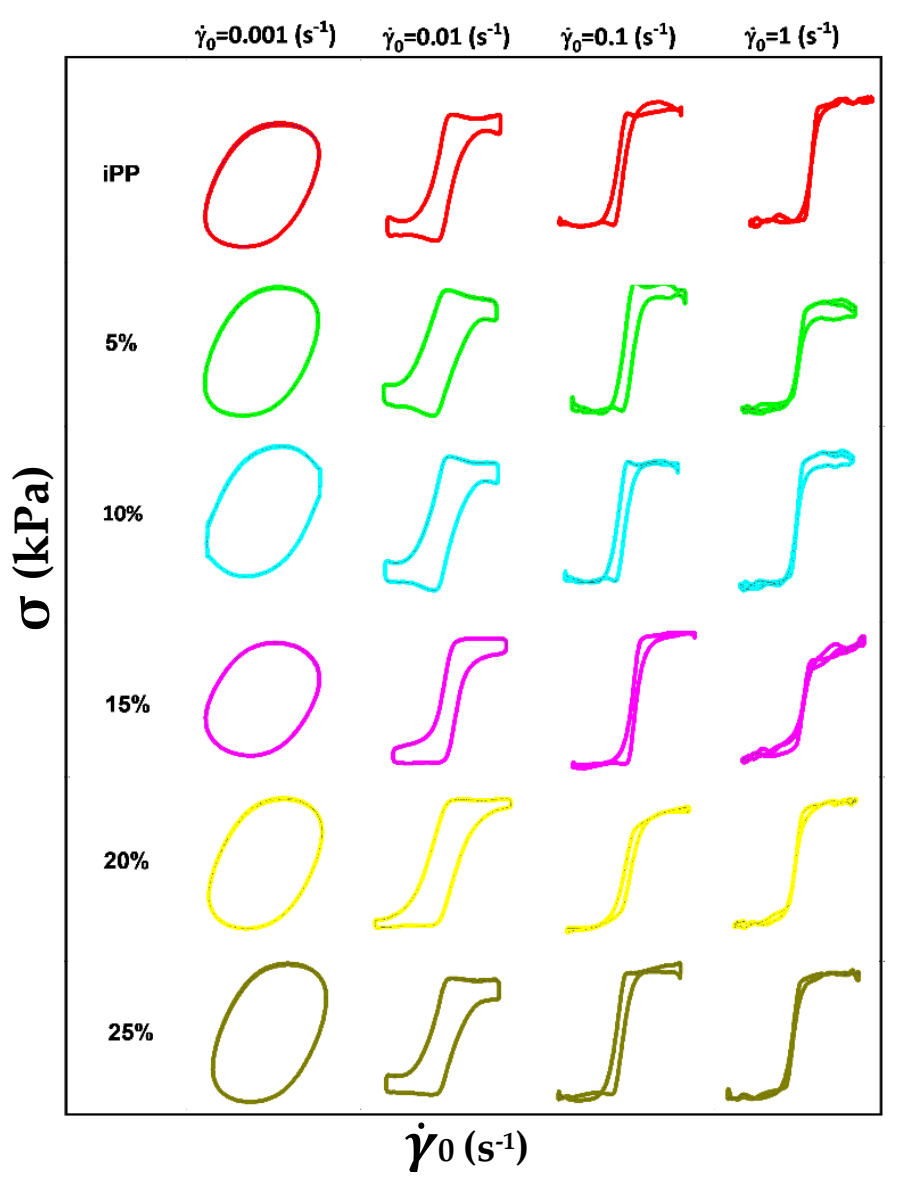

(b)

Figure 5. Lissajous curve plots of dynamic stress $(\sigma)$ against (a) dynamic strain $\left(\gamma_{0}\right)$ and (b) strain-rate amplitude $\left(\dot{\gamma}_{0}\right)$ for pure iPP, LMPP and blends at $1.0 \mathrm{rad} \mathrm{s}^{-1}$.

In Figure 6, Fourier transform spectrum shows odd high-order harmonics for nonlinearity from dominant responses of the first harmonic in all samples. From the relative intensities $\left(\mathrm{I}_{\mathrm{n}} / \mathrm{I}_{1}\right)$, the values of $\mathrm{I}_{3} / \mathrm{I}_{1}, \mathrm{I}_{5} / \mathrm{I}_{1}$ and $\mathrm{I}_{7} / \mathrm{I}_{1}$ in the blends reduce at lower $\gamma_{0}$, compared to pure iPP. Blends with 15, 20 and 25\% LMPP contents showed slightly higher intensities than pure iPP (Figure 6b), indicating LMPP affects viscoelasticity nonlinearities.

Under LAOS stresses, the blends demonstrated subtle large-strain elastic modulus $\left(G_{L}\right)$ and large strain-rate dynamic viscosity $\left(\eta_{L}\right)$, while minimum-strain elastic modulus $\left(G_{M}\right)$ and minimum strain-rate dynamic viscosity $\left(\eta_{M}\right)$ were influenced with the addition of LMPP as shown in Figure 7. Dependence of $\mathrm{G}_{\mathrm{L}}$ and $\eta_{\mathrm{L}}$ with increasing $\gamma_{0}$ and $\dot{\gamma}_{0}$ in linear to nonlinearity regime was obvious, while $G_{M}$ and $\eta_{M}$ were dominant in linear regime. With both $G_{L}$ and $G_{M}$ decreasing with increasing $\gamma_{0}$, the blends and pure iPP exhibit overall intercycle strain softening. Whereas decreasing $\eta_{L}$ and $\eta_{M}$ showed intercycle shear thinning behavior in blends and pure iPP.

In Figure 8, the intracycle and intercycle nonlinear responses were quantified as strainstiffening ratio $(S=1-G M / G L)$ and shear thickening ratio $(T=1-\eta M / \eta L)[14,35]$, where blends showed arbitrary behavior at higher $\gamma_{0}$, similarly with subtle $Q$ parameter. It is a ratio of the intensity of the 3rd harmonic response of elastic stress to the 1 st $\left(\mathrm{I}_{3 / 1}\right)$, divided by the strain amplitude squared $\gamma_{0}^{2}$, calculated as $\mathrm{Q}\left(\omega, \gamma_{0}\right) \equiv \mathrm{I}_{3 / 1} / \gamma_{0}^{2}$ [36], providing information on polymer systems with two material interfaces [37]. Such intrinsic nonlinear parameters have been used to assess dispersion/distribution, polymeric interface interactions in blends and quantification of blends mixing [36-38]. 


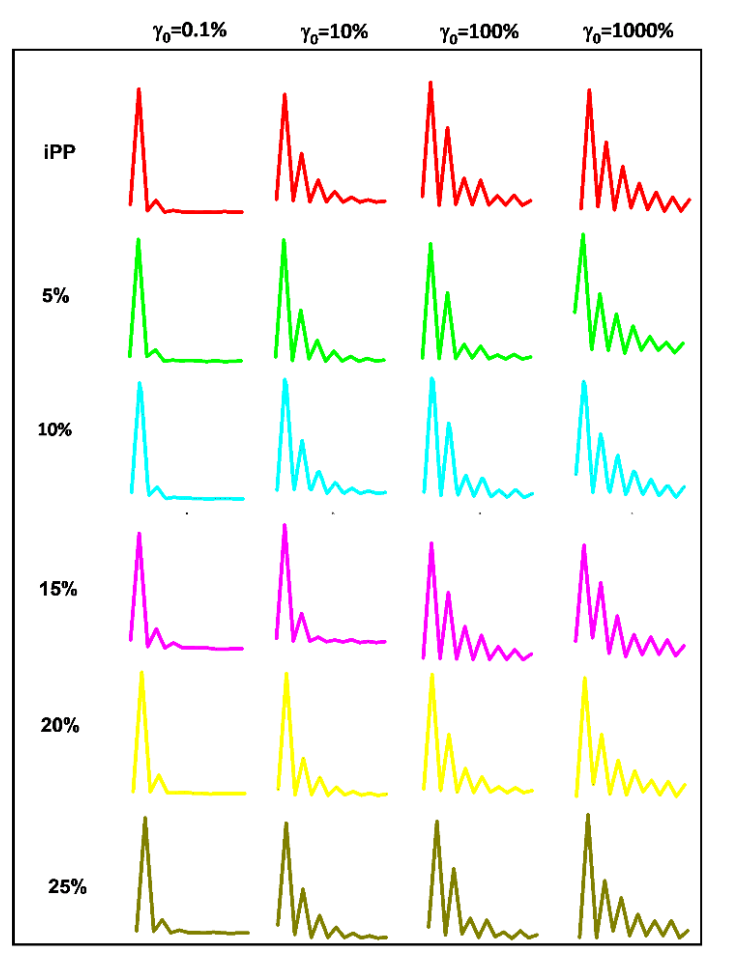

Fourier harmonics [n]

(a)

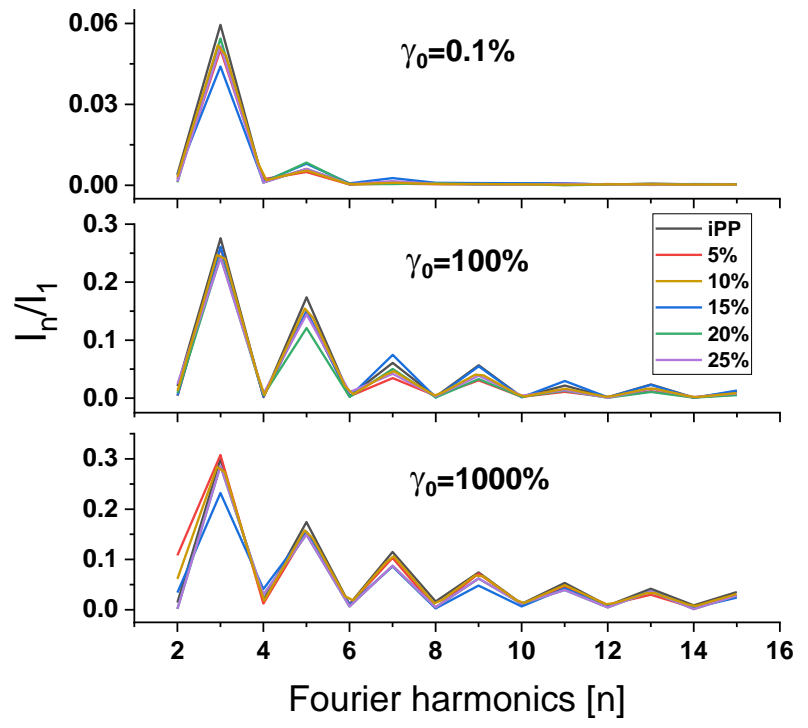

(b)

Figure 6. Fourier spectra at different dynamic strains $\left(\gamma_{0}\right)$ for pure iPP, LMPP and blends at $1.0 \mathrm{rad} \mathrm{s}^{-1}$ (a), (b) showing comparative intensities of blends at given $\gamma_{0}$.
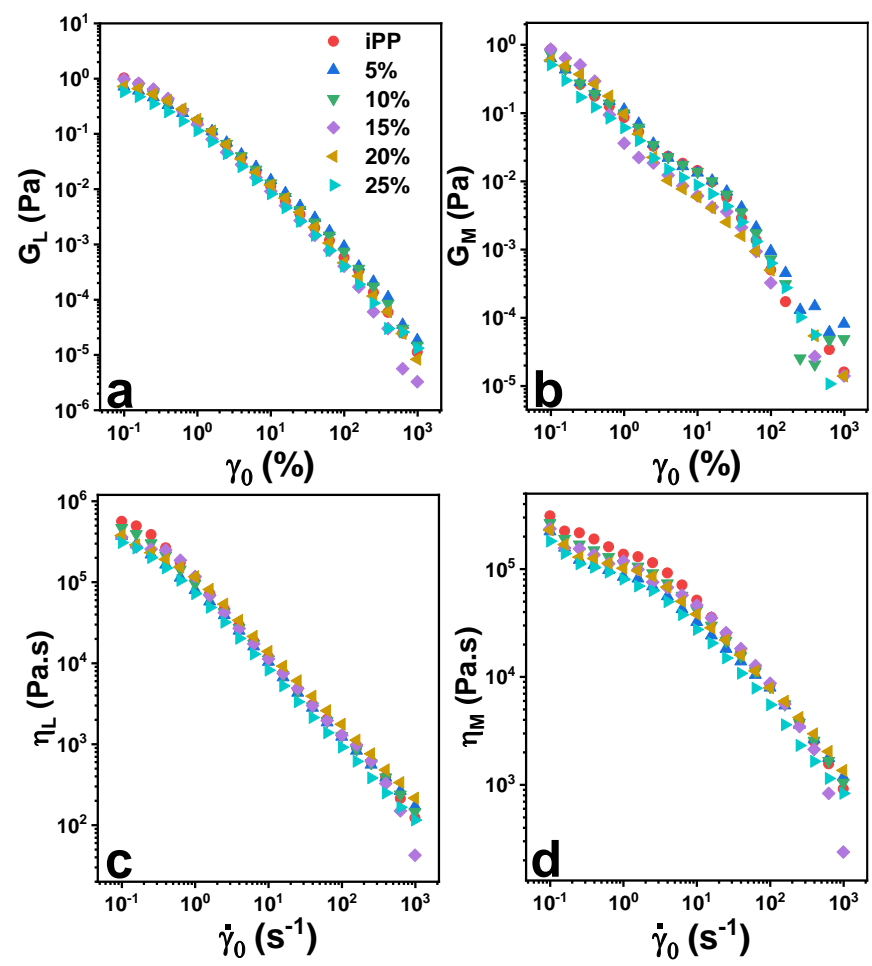

Figure 7. Large-strain elastic modulus $\left(\mathrm{G}_{\mathrm{L}} ;(\mathbf{a})\right)$, minimum-strain elastic modulus $\left(\mathrm{G}_{\mathrm{M}} ;(\mathbf{b})\right)$, large strain-rate dynamic viscosity $\left(\eta_{L} ;(\mathbf{c})\right)$ and minimum strain-rate dynamic viscosity $\left(\eta_{M} ;(d)\right)$ as a function of strain-rate amplitude $\dot{\gamma}_{0}$ and strain amplitude $\gamma_{0}$ under LAOS stress for pure iPP, LMPP and blends at $1.0 \mathrm{rad} \mathrm{s}^{-1}$. 

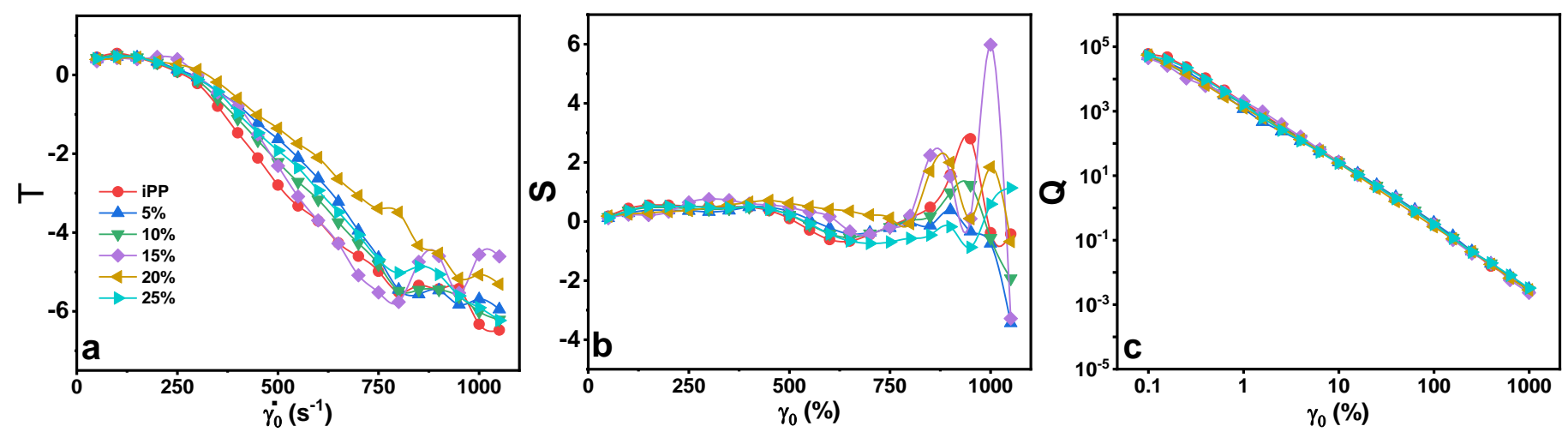

Figure 8. Intracycle strain thickening $(\mathrm{T} ;(\mathbf{a}))$, intracycle strain stiffening $(\mathrm{S} ;(\mathbf{b}))$ and $\mathrm{Q}$ parameter $(\mathbf{c})$ as a function of strain-rate amplitude $\dot{\gamma}_{0}$ and strain amplitude $\gamma_{0}$ under LAOS stress for pure iPP, LMPP and blends at $1.0 \mathrm{rad} \mathrm{s}^{-1}$.

\section{Discussion}

The spherulite shape and size are the obvious characteristics [39]. Spherulitic growth rate is related to the crystallization temperature and is nonlinear in non-isothermal conditions of the polymeric blends. The POM shown in Figure 1, the spherulitic growth rate of the blends presents regular growth with the addition of LMPP contents, affecting the size and shape of the spherulites. With the addition of LMPP, there is a change in the crystallization temperature of the blends, affecting the melt viscosity, crystal diffusion rate of the chains and their segmental mobility (Figure 2) [40]. LMPP is a low tacticity polypropylene, known for great spinability and elongation [41]. In our previous study, the blends with $5 \%, 10 \%$ and $15 \%$ LMPP contents showed crystallinity at $43.2 \%, 43.9 \%$ and $42.5 \%$, while the numbers were $40.2 \%$ and $16.5 \%$ for pure iPP and LMPP. Similarly, the mid content ratios showed enhanced mechanical properties, while showing the highest elongation for 25\% LMPP blend [3]. Overview of crystallinity and thermal properties of the blends is given in Table 2, calculated previously [3] from differential scanning calorimeter (DSC) and X-ray diffraction (XRD). From DSC analysis, the crystallization temperature $(\mathrm{tC})$ of blends decreases with increased LMPP content, while the tM and melt enthalpy $\left(\triangle \mathrm{H}_{\mathrm{f}}\right)$ of pure LMPP is lower, indicating low crystallinity and different crystal type from iPP [3,7]. Whereas the average spherulite size of blends appeared enlarged with the LMPP addition at lower contents $(\sim 61 \mu \mathrm{m})$, this later reduces at higher contents significantly $(\sim 52 \mu \mathrm{m})$.

Table 2. Crystallinity and thermal properties of the blends. Data from [3].

\begin{tabular}{cccccc}
\hline iPP/ LMPP & ${ }^{\mathbf{1}} \mathbf{T C}\left({ }^{\circ} \mathbf{C}\right)$ & $\mathbf{T M}\left({ }^{\circ} \mathbf{C}\right)$ & ${ }^{\mathbf{2}} \triangle \mathbf{H}_{\mathbf{f}}(\mathbf{J} / \mathbf{g})$ & Crystallinity $(\%)$ & Spherulite Size $(\boldsymbol{\mu m})$ \\
\hline $100 / 0$ & 116.3 & 159.1 & 91.0 & 40.2 & 50 \\
$0 / 100$ & 40.1 & 79.1 & 20.6 & 16.5 & 0 \\
$95 / 5$ & 116.0 & 158.2 & 84.2 & 43.2 & 58 \\
$90 / 10$ & 115.8 & 159.1 & 77.0 & 40.9 & 61 \\
$85 / 15$ & 115.3 & 157.4 & 72.1 & 42.5 & 60 \\
$80 / 20$ & 114.2 & 156.5 & 68.2 & 38.8 & 52 \\
$75 / 25$ & 114.0 & 155.3 & 65.0 & 38.3 & \\
\hline
\end{tabular}

${ }^{1}$ TC: Crystallization temperature; ${ }^{2} \triangle \mathrm{H}_{\mathrm{f}}$ : Melt enthalpy.

It is demonstrated that the shear viscosity is stable at given temperatures, with increased LMPP content, keeping the rheological flow (capillary rheology) preserved similar to iPP, as shown in Figure 3a-c. This suggests that addition of LMPP in iPP blends will not affect processing extrusion or injection molding. With improved chain mobility the iPP/LMPP melts undergo low shear viscosity at all given temperatures without any flow resistance. It can be deduced that pure iPP and its blends with LMPP demonstrate shearthinning behavior.

In the linear rheology, the $\omega$ dependent linear $G^{\prime}$ and $G^{\prime \prime}$ in Figure 4, with initial dominant $G^{\prime}>G^{\prime \prime}$ and slight $G^{\prime}<G^{\prime \prime}$ at higher $\omega$ in all the blends, structural relaxation 
was demonstrated. Figure 5 describes the viscoelastic perspective of the materials, which is difficult to distinguish from the $G^{\prime}$ and $G^{\prime \prime}$ only. From the qualitative information from LAOS rheological behavior, the blends showed enhanced nonlinearities with the addition of LMPP. The ascending addition of LMPP to the blends does not significantly influence the shape compared to pure iPP, indicating the stabilized structure. However, the blends at variant $\gamma_{0}$ and $\dot{\gamma}_{0}$ showed sharper edged Lissajous figures, with slightly smoother final shapes. Such changes are associated with the enhanced dissipation of the mechanical energy within a cycle [42]. From the Fourier transform rheology in Figure 6, the higherorder odd harmonics for relative intensities $\left(\mathrm{I}_{\mathrm{n}} / \mathrm{I}_{1}\right)$ are normalized by the fundamental one; here however, the affected viscoelasticity nonlinearity of the blends may relate to but not be determined by the amount of LMPP.

For the nonlinear viscoelastic moduli shown in Figure 7, all samples may demonstrate shear-thickening trend; however, blend responses were sensitive when it comes to $\eta_{M}$ and $\mathrm{G}_{\mathrm{M}}$, yielding a LAOS fingerprint. The LAOS fingerprinting is used to define specific viscoelastic nonlinearities [27]. The blends showed primarily elastic nonlinearities, however; affected $\eta_{M}$ at lower $\dot{\gamma}_{0}$, samples showed predominantly strain-rate softening behavior.

From Figure 8, the elastic nonlinearity perspective of the blends in initial to medium $\gamma_{0}$ showed weak intracycle softening $(S<0)$, while showing intracycle hardening $(S>0)$ at higher $\gamma_{0}$ range, where 20 and $25 \%$ predominate compared to pure iPP. The blends' behavior towards viscous nonlinearity was less arbitrary, where all blends and pure iPP featured shear thinning $(\mathrm{T}<0)$ at medium to high $\dot{\gamma}_{0}$. This showed dependence of the intracycle viscoelastic behavior with regards to dimensionless but prominent $S$ nonlinearity in high-content blends compared to $\mathrm{T}$, where there was a gradual decline.

\section{Conclusions}

In accordance with the previous study, degree of crystallinity of blends increases for $5-15 \%$ LMPP contents and then progressively decreases for $20-25 \%$. Similarly, the addition of LMPP in iPP blends affects the spherulitic growth rate in size and shape with clear boundaries and bright light birefringence. This spherulitic effect was apparent in high-content LMPP blends, which also reduces the crystal nucleus size and promotes molecular dispersion/distribution in iPP. In terms of responses from capillary rheology, shear-thinning behavior was observed in pure iPP and all blends. The dynamic linear rheological responses were subtle in all specimens, whereas variant intrinsic viscoelastic nonlinearities were observed from LAOS tests. High-content LMPP blends posed intracycle strain-thickening and intracycle strain-stiffening behavior at medium strain amplitudes. From this LAOS study, change in the viscoelastic nonlinearities of iPP blended with LMPP was observed in both, strain and strain-rate space.

Author Contributions: Conceptualization, F.Z.; S.Y. and M.H.; methodology, F.Z.; S.Y. and M.H.; software, S.Y. and M.H.; validation, F.Z.; S.Y. and M.H.; formal analysis, F.Z.; S.Y. and M.H..; investigation F.Z.; S.Y. and M.H.; resources F.Z.; S.Y. and M.H.; data curation, S.Y. and M.H.; writing-original draft preparation, F.Z.; S.Y. and M.H.; writing—review and editing, F.Z.; S.Y. and M.H.; visualization, S.Y. and M.H.; supervision, S.Y. All authors have read and agreed to the published version of the manuscript.

Funding: Natural Science Foundation of Zhejiang Province (Grant number LQ21E030013) and Scientific Foundation of Zhejiang Sci-Tech University (Grant number 20202293-Y, 2020YBZX11).

Institutional Review Board Statement: Not applicable.

Informed Consent Statement: Not applicable.

Data Availability Statement: The data presented in this study are available upon request from the corresponding author.

Conflicts of Interest: The authors declare no conflict of interest. 


\section{References}

1. Nakatani, A.I.; Zhang, S.; Ozair, S.; Peera, A.; Young, W.O.; Kennedy, K.M.; Peterson, C.; Pearson, C.; Ray, H. Relationship between Large Amplitude Oscillatory Shear (LAOS) Experiments and Commercial Pressure Sensitive Adhesives Applications Testing. Int. J. Adhes. Adhes. 2021, 108, 102869. [CrossRef]

2. Kanai, T.; Kohri, Y.; Takebe, T. Theoretical Analysis of the Spunbond Process and Its Applications for Polypropylenes. In AIP Conference Proceedings; AIP Publishing: New York, NY, USA, 2016; Volume 1779, p. 120001.

3. Hussain, M.; Zhu, F.; Zhu, F.; Yu, B.; Yu, B.; Han, J.; Han, J.; Memon, H.; Yasin, S. LMPP Effects on Morphology, Crystallization, Thermal and Mechanical Properties of IPP/LMPP Blend Fibres. Fibres Text. East. Eur. 2018, 26, 26-31. [CrossRef]

4. Yasin, S.; Sun, D.; Memon, H.; Zhu, F.; Jian, H.; Bin, Y.; Mingbo, M.; Hussain, M. Optimization of Mechanical and Thermal Properties of IPP and LMPP Blend Fibres by Surface Response Methodology. Polymers 2018, 10, 1135. [CrossRef] [PubMed]

5. Okazaki, G.; Secrist, K.E.; Gray, S.D. Hot Melt Adhesives for Bonding Elastomeric Components, Nonwoven Materials, and Thermoplastic Films. U.S. Patent US20180148616A1, 31 May 2018.

6. Robledo, N.; Vega, J.F.; Nieto, J.; Martínez-Salazar, J. Role of the Interface in the Melt-Rheology Properties of Linear Low-Density Polyethylene/Low-Density Polyethylene Blends: Effect of the Molecular Architecture of the Dispersed Phase. J. Appl. Polym. Sci. 2011, 119, 3217-3226. [CrossRef]

7. Gray, S.D.; Freund, D.F.; Hamann, R.E.; Hu, M.; Flores, F.N.-H. Polyolefin-Based Hot Melt Adhesives with Improved Processing And Bonding Performance. U.S. Patent US20160102230A1, 14 April 2016.

8. Martuscelli, E.; Silvestre, C.; Abate, G. Morphology, Crystallization and Melting Behaviour of Films of Isotactic Polypropylene Blended with Ethylene-Propylene Copolymers and Polyisobutylene. Polymer 1982, 23, 229-237. [CrossRef]

9. Sadiku-Agboola, O.; Sadiku, R.E.; Adegbola, A.T.; Biotidara, O.F. Rheological Properties of Polymers: Structure and Morphology of Molten Polymer Blends. Mater. Sci. Appl. 2011, 2, 30-41. [CrossRef]

10. Hammani, S.; Moulai-Mostefa, N.; Samyn, P.; Bechelany, M.; Dufresne, A.; Barhoum, A. Morphology, Rheology and Crystallization in Relation to the Viscosity Ratio of Polystyrene/Polypropylene Polymer Blends. Materials 2020, 13, 926. [CrossRef]

11. Ariff, Z.; Ariffin, A.; Jikan, S.; Abdul Rahim, N. Rheological Behaviour of Polypropylene through Extrusion and Capillary Rheometry. In Polypropylene; IntechOpen: London, UK, 2012; pp. 29-48.

12. Ariffin, A.; Jikan, S.S.; Samsudin, M.S.F.; Ariff, Z.M.; Ishak, Z.A.M. Melt Elasticity Phenomenon of Multicomponent (Talc and Calcium Carbonate) Filled Polypropylene. J. Reinf. Plast. Compos. 2006, 25, 913-923. [CrossRef]

13. Zhu, F.; Su, J.; Zhao, Y.; Hussain, M.; Yasin, S.; Yu, B.; Han, J. Influence of Halloysite Nanotubes on Poly(Lactic Acid) Melt-Blown Nonwovens Compatibilized by Dual-Monomer Melt-Grafted Poly(Lactic Acid). Text. Res. J. 2019, 89, 4173-4185. [CrossRef]

14. Trebar, M.; Lotrič, U. Predicting Mechanical Properties of Rubber Compounds with Neural Networks and Support Vector Machines. In Proceedings of the ICANNGA 2007: 8th International Conference on Adaptive and Natural Computing Algorithms, Warsaw, Poland, 11-14 April 2007; Beliczynski, B., Dzielinski, A., Iwanowski, M., Ribeiro, B., Eds.; Springer: Berlin/Heidelberg, Germany, 2007; pp. 254-261.

15. Yasin, S.; Hussain, M.; Zheng, Q.; Song, Y. Large Amplitude Oscillatory Rheology of Silica and Cellulose Nanocrystals Filled Natural Rubber Compounds. J. Colloid Interface Sci. 2020. [CrossRef]

16. Yasin, S.; Hussain, M.; Zheng, Q.; Song, Y. Effects of Ionic Liquid on Cellulosic Nanofiller Filled Natural Rubber Bionanocomposites. J. Colloid Interface Sci. 2021, 591, 409-417. [CrossRef]

17. Stephen, R.; Alex, R.; Cherian, T.; Varghese, S.; Joseph, K.; Thomas, S. Rheological Behavior of Nanocomposites of Natural Rubber and Carboxylated Styrene Butadiene Rubber Latices and Their Blends. J. Appl. Polym. Sci. 2006, 101, 2355-2362. [CrossRef]

18. Vijayan, P.P.; Puglia, D.; Kenny, J.M.; Thomas, S. Effect of Organically Modified Nanoclay on the Miscibility, Rheology, Morphology and Properties of Epoxy/Carboxyl-Terminated (Butadiene-Co-Acrylonitrile) Blend. Soft Matter 2013, 9, 2899-2911. [CrossRef]

19. Schlatter, G.; Fleury, G.; Muller, R. Fourier Transform Rheology of Branched Polyethylene: Experiments and Models for Assessing the Macromolecular Architecture. Macromolecules 2005, 38, 6492-6503. [CrossRef]

20. Cho, K.S.; Hyun, K.; Ahn, K.H.; Lee, S.J. A Geometrical Interpretation of Large Amplitude Oscillatory Shear Response. J. Rheol. 2005, 49, 747-758. [CrossRef]

21. Kallus, S.; Willenbacher, N.; Kirsch, S.; Distler, D.; Neidhöfer, T.; Wilhelm, M.; Spiess, H.W. Characterization of Polymer Dispersions by Fourier Transform Rheology. Rheol. Acta 2001, 40, 552-559. [CrossRef]

22. Wilhelm, M.; Reinheimer, P.; Ortseifer, M.; Neidhöfer, T.; Spiess, H.-W. The Crossover between Linear and Non-Linear Mechanical Behaviour in Polymer Solutions as Detected by Fourier-Transform Rheology. Rheol. Acta 2000, 39, 241-246. [CrossRef]

23. Ewoldt, R.H.; Winter, P.; Maxey, J.; McKinley, G.H. Large Amplitude Oscillatory Shear of Pseudoplastic and Elastoviscoplastic Materials. Rheol. Acta 2010, 49, 191-212. [CrossRef]

24. Shamim, N.; McKenna, G.B. Mechanical Spectral Hole Burning in Polymer Solutions: Comparison with Large Amplitude Oscillatory Shear Fingerprinting. J. Rheol. 2014, 58, 43-62. [CrossRef]

25. Wilhelm, M. Fourier-Transform Rheology. Macromol. Mater. Eng. 2002, 287, 83-105. [CrossRef]

26. Nam, J.G.; Ahn, K.H.; Lee, S.J.; Hyun, K. First Normal Stress Difference of Entangled Polymer Solutions in Large Amplitude Oscillatory Shear Flow. J. Rheol. 2010, 54, 1243-1266. [CrossRef]

27. Vishal, B.; Ghosh, P. Nonlinear Viscoelastic Behavior of Aqueous Foam under Large Amplitude Oscillatory Shear Flow. Korea-Aust. Rheol. J. 2018, 30, 147-159. [CrossRef] 
28. Ewoldt, R.H.; Hosoi, A.E.; McKinley, G.H. New Measures for Characterizing Nonlinear Viscoelasticity in Large Amplitude Oscillatory Shear. J. Rheol. 2008, 52, 1427-1458. [CrossRef]

29. Hussain, M.; Zhu, F.; Yu., B.; Han, J.; Du, Z. Study on enhancing the strength and toughness of isotactic polypropylene spunbonded nonwovens by low-modulus polypropylene. J. Zhejiang Sci-Tech Univ. Nat. Sci. 2016, 35, 803-807.

30. Chen, J.-H.; Tsai, F.-C.; Nien, Y.-H.; Yeh, P.-H. Isothermal Crystallization of Isotactic Polypropylene Blended with Low Molecular Weight Atactic Polypropylene. Part I. Thermal Properties and Morphology Development. Polymer 2005, 46, 5680-5688. [CrossRef]

31. Liu, Y.; Tian, B.; Liu, X. Effect of Interaction Enhancement on Rheological Response of Polypropylene/Polybutadiene Blend Composites. Polym. Test. 2021, 96, 107069. [CrossRef]

32. Hussain, M.; Yasin, S.; Adnan Akram, M.; Xu, H.; Song, Y.; Zheng, Q. Influence of Ionic Liquids on Structure and Rheological Behaviors of Silica-Filled Butadiene Rubber. Ind. Eng. Chem. Res. 2019, 58, 18205-18212. [CrossRef]

33. Hussain, M.; Yasin, S.; Memon, H.; Li, Z.; Fan, X.; Akram, M.A.; Wang, W.; Song, Y.; Zheng, Q. Rheological and Mechanical Properties of Silica/Nitrile Butadiene Rubber Vulcanizates with Eco-Friendly Ionic Liquid. Polymers 2020, 12, 2763. [CrossRef]

34. Zare, Y.; Park, S.P.; Rhee, K.Y. Analysis of Complex Viscosity and Shear Thinning Behavior in Poly (Lactic Acid)/Poly (Ethylene Oxide)/Carbon Nanotubes Biosensor Based on Carreau-Yasuda Model. Results Phys. 2019, 13, 102245. [CrossRef]

35. Kim, J.; Merger, D.; Wilhelm, M.; Helgeson, M.E. Microstructure and Nonlinear Signatures of Yielding in a Heterogeneous Colloidal Gel under Large Amplitude Oscillatory Shear. J. Rheol. 2014, 58, 1359-1390. [CrossRef]

36. Hyun, K.; Wilhelm, M.; Klein, C.O.; Cho, K.S.; Nam, J.G.; Ahn, K.H.; Lee, S.J.; Ewoldt, R.H.; McKinley, G.H. A Review of Nonlinear Oscillatory Shear Tests: Analysis and Application of Large Amplitude Oscillatory Shear (LAOS). Prog. Polym. Sci. 2011, 36, 1697-1753. [CrossRef]

37. Hyun, K.; Wilhelm, M. Establishing a New Mechanical Nonlinear Coefficient Q from FT-Rheology: First Investigation of Entangled Linear and Comb Polymer Model Systems. Macromolecules 2009, 42, 411-422. [CrossRef]

38. Hyun, K.; Kim, W. A New Non-Linear Parameter Q from FT-Rheology under Nonlinear Dynamic Oscillatory Shear for Polymer Melts System. Korea-Aust. Rheol. J. 2011, 23, 227-235. [CrossRef]

39. Ellingham, T.; Yilmaz, G.; Turng, L.-S. Non-Linear Rheological Response as a Tool for Assessing Dispersion in Polypropylene/Polycaprolactone/Clay Nanocomposites and Blends Made with Sub-Critical Gas-Assisted Processing. Polym. Eng. Sci. 2020, 60, 55-60. [CrossRef]

40. Hemsley, D. 33-Optical Microscopy. In Comprehensive Polymer Science and Supplements; Allen, G., Bevington, J.C., Eds.; Pergamon: Amsterdam, The Netherlands, 1989; pp. 765-784. ISBN 978-0-08-096701-1.

41. Wang, B.; Lin, F.; Li, X.; Zhang, Z.; Xue, X.; Liu, S.; Ji, X.; Yu, Q.; Yuan, Z.; Chen, X.; et al. Isothermal Crystallization and Rheology Properties of Isotactic Polypropylene/Bacterial Cellulose Composite. Polymers 2018, 10, 1284. [CrossRef]

42. Idemitsu Kosan. New Polyolefin LMPO for Elastic Nonwovens. Chem. Fibers Int. 2008, 58, 90. 